\title{
Efficacy of sulfadoxine/ pyrimethamine for uncomplicated Plasmodium falciparum malaria in a small sample of Sudanese children
}

I. Adam, ${ }^{1}$ M.H. Ibrahim, ${ }^{1}$ I.A. Alelbasit ${ }^{2}$ and M.I. Elbashir ${ }^{2}$

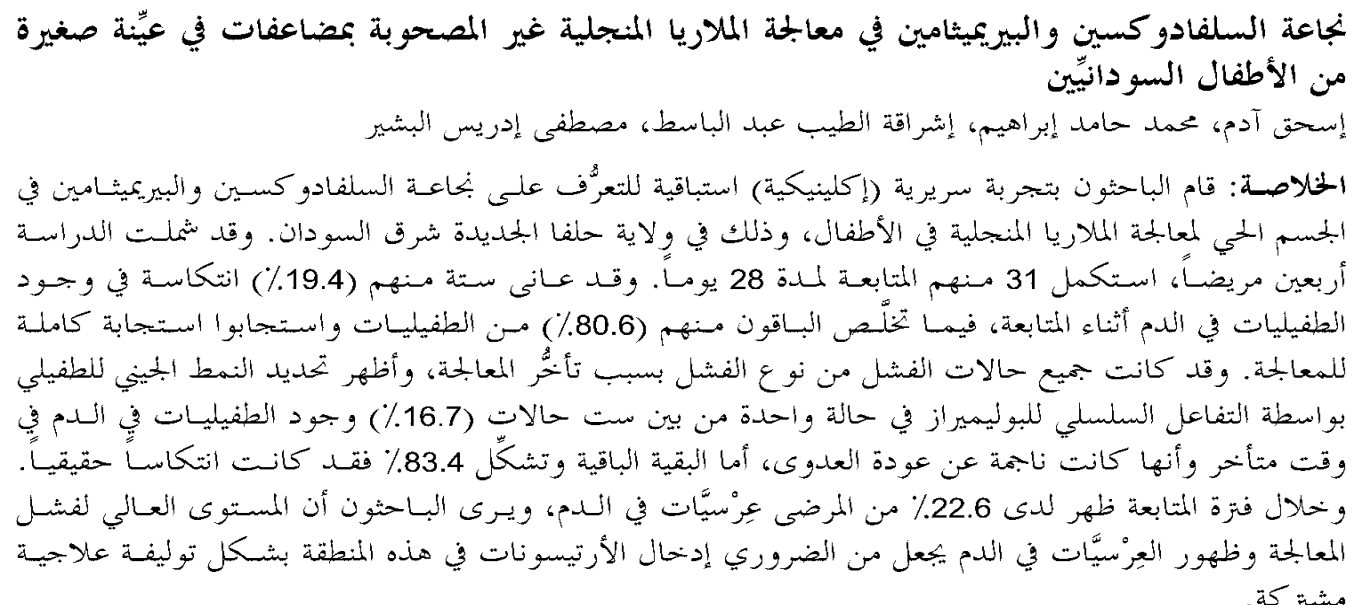

ABSTRACT A prospective clinical trial was carried out to determine in vivo efficacy of sulfadoxine/pyrimethamine for the treatment of uncomplicated Plasmodium falciparum malaria in children in New Halfa. Forty patients were enrolled; 31 completed the 28-day follow-up. Six (19.4\%) patients showed recurrence of parasitaemia during follow-up, while the rest $(80.6 \%)$ cleared the parasites and responded fully to treatment. All the failures were late treatment failures. Parasite genotyping showed that $1(16.7 \%)$ of the 6 cases of late parasitaemia was due to reinfection while the rest $(83.4 \%)$ were due to true recrudescence. During the follow-up period $22.6 \%$ of patients showed gametocytaemia. The high level of treatment failure as well as gametocytaemia necessitates the introduction of artesunate in this area in combination therapy.

Efficacité de la sulfadoxine-pyriméthamine pour le paludisme à Plasmodium falciparum sans complications dans un petit échantillon d'enfants soudanais

RESUME Un essai clinique prospectif a été effectué afin de déterminer l'efficacité in vivo de l'association sulfadoxine-pyriméthamine dans le traitement de l'accès palustre simple à Plasmodium falciparum chez des enfants à New Halfa. Quarante patients ont été recrutés ; 31 ont terminé la période de suivi de 28 jours. Six patients $(19,4 \%)$ ont présenté une parasitémie récurrente pendant le suivi, tandis que le reste des patients $(80,6 \%)$ ont éliminé les parasites et ont répondu au traitement complètement. Tous les échecs étaient des échecs thérapeutiques tardifs. Le génotypage du parasite a montré qu'un cas sur 6 (16,7\%) des parasitémies tardives était dû à une réinfestation alors que le reste $(83,4 \%)$ était dû à une recrudescence. Pendant la période de suivi, 22,6\% des patients ont présenté une gamétocytémie. Le niveau élevé d'échec thérapeutique ainsi que la gamétocytémie nécessitent l'introduction de l'artésunate dans cette région en traitement associé.

${ }^{1}$ New Halfa Hospital, New Halfa, Sudan.

${ }^{2}$ Faculty of Medicine, University of Khartoum, Khartoum, Sudan.

Received: 24/06/03; accepted:29/09/03

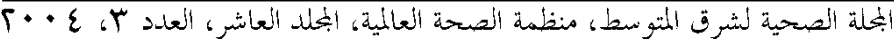




\section{Introduction}

There are around 765000 childhood deaths in Africa due to Plasmodium falciparum infections every year [1]. Children are at higher risk for developing drug-resistant falciparum malaria than adults $[2,3]$. Countries with high levels of resistance have witnessed increased childhood morbidity and mortality [4]. Due to widespread resistance to chloroquine treatment, several African countries have changed their policy from chloroquine as first-line treatment to sulfadoxine/pyrimethamine. In Sudan, all grades of chloroquine resistance were reported in children, where $25 \%$ of the parasite isolates were chloroquine resistant; $16.7 \%$ were the severe form of resistance [5]. Recently, chloroquine resistance was reported to be over $75 \%$ in the capital and eastern Sudan $[6,7]$.

Despite these reports, chloroquine is still the first-line treatment for uncomplicated falciparum malaria in Sudan, while sulfadoxine/pyrimethamine is the secondline treatment. Resistance to sulfadoxine/ pyrimethamine was first reported in central Sudan in 1992 [8]. Thereafter much of the data are confusing; in 1 study $10.7 \%$ resistance was reported [9], while in 2 other studies carried out in eastern Sudan 100\% sensitivity was reported by in vivo and in vitro approaches $[6,10]$. To address the issue further and to obtain data on children we conducted a study on a small sample of Sudanese children.

\section{Methods}

\section{Study area}

The study was conducted at the Elhara Eloula health centre in the New Halfa area, eastern Sudan, during November 2001 to January 2002. New Halfa is an agricultural area, $500 \mathrm{~km}$ from Khartoum. It is $450 \mathrm{~m}$ above sea level and is characterized by an average annual rainfall of $238 \mathrm{~mm}$ and average annual relative humidity of $35 \%$.

The area is mesoendemic for malaria with the peak during the rainy and postrainy season (September to January) [11]. The predominant malaria species is $P$. falciparum, with $P$. vivax and $P$. malariae occasionally seen [6]. Anopheles arabiensis is the sole malaria vector in the area [12]. Bednets and chemoprophylaxis are not used in the area.

\section{Patients}

Children with documented axillary temperature $\geq 37.5^{\circ} \mathrm{C}$ and P. falciparum infection were eligible for inclusion if their parents or guardian gave informed consent. Children were excluded if they had 1 or more manifestations of severe malaria [13], concurrent infection, known allergy to sulfonamides, or treatment within the last 2 weeks with a sulfonamide, quinine or mefloquine. History of chloroquine treatment was not an exclusion criterion.

A complete history of all enrolled children was taken and they all underwent a thorough physical examination by the medical officer and received sulfadoxine/ pyrimethamine (sulfadoxine $25 \mathrm{mg} / \mathrm{kg}$, Amipharma laboratory, Sudan). The children were given the medication orally under supervision and monitored for 30 minutes. A second full dose was administered if the child vomited. Children who vomited the drug for the second time were excluded from the study and were given parenteral quinine.

\section{Investigations}

Using finger-prick blood samples, thick and thin blood smears were prepared from each patient, stained with Giemsa ( $\mathrm{pH}$ 7.0, diluted in phosphate-buffered saline) and counted against 200 white blood cells as-

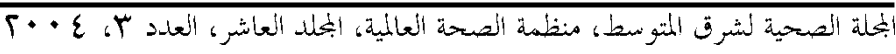


suming that the number of white blood cells is $6000 / \mathrm{mm}^{3}$ of blood. Trophozoites and gametocytes of $P$. falciparum were recorded separately. The blood films were prepared and examined by a highly expert technician and cross-checked by another technician blinded to the results and verified by the supervisor if there was any disagreement. Thin-film blood samples, fixed in methanol and Giemsa-stained were prepared when the parasite species was doubtful. The blood films were repeated on days $1,2,3,7,14,21$ and 28 or at any time if symptoms recurred.

Three spots of blood were taken on filter paper initially and later if parasites reappeared microscopically during the followup period. Primers from 3 polymorphic $P$. falciparum antigens - merozoite surface protein-1 and -2 and glutamate-rich protein-were used in polymerase chain reaction (PCR) analysis to differentiate between reinfection and recrudescence [14].

\section{Follow-up}

Parents were asked to bring the children to the health centre for follow-up on days 1 , $2,3,7,14,21$ and 28 or if they developed febrile symptoms and felt unwell. Parents were asked about the presence of fever, vomiting and diarrhoea. At each visit, a brief physical examination, including axillary temperature, was performed and blood was taken for thick-film tests.

\section{Outcome measures}

The 28-day efficacy of sulfadoxine/pyrimethamine was assessed by a modified World Health Organization protocol for uncomplicated falciparum malaria for areas of moderate or low malaria transmission [15]. Patients were classified as early treatment failure if they developed dangerous signs or severe malaria on day 3 , the parasite density on day 2 exceeded that on day 0 , or the parasite density on day 3 was $\geq 25 \%$ of that on day 0 . Late treatment failure was defined as the development of symptoms or signs of severe malaria, or development of any parasitaemia with or without fever, after day 3. All other cases were regarded as adequate clinical and parasitological response. Those with treatment failure were given quinine $10 \mathrm{mg} / \mathrm{kg}$ for 7 days.

Data were entered into a microcomputer using SPSS/PC batching for data analysis. Simple frequencies, percentages, means and standard deviation (SD) were calculated. The data of the patients with recurrent parasitaemia were compared with those without recurrence by Student $t$-test, chi-squared and Fisher exact test when applicable; $P \leq 0.05$ was regarded as significant.

\section{Results}

Thirty-one out of 40 patients completed the study. The others were excluded due to loss to follow-up or change of address (5), contracting another disease (2) or self-use of other treatments (2).

Table 1 shows the presenting characteristics of the 31 patients. The mean $\pm \mathrm{SD}$ age was $6.9 \pm 3.9$ years, range $1-14$ years. The age of $12 / 31$ patients $(38.7 \%)$ was below 5 years. All patients were aparasitaemic and only $3 / 31$ patients $(9.7 \%)$ were febrile on day 3 .

Twenty-five out of 31 patients $(80.6 \%)$ cleared the parasites and responded fully to treatment. In 6 patients $(19.4 \%)$ the parasitaemia recurred during follow-up (2/6 on day $7,1 / 6$ on day $14,2 / 6$ on day 21 and $1 / 6$ on day 28) and they were defined as late treatment failures. Reinfection was confirmed in 1 patient (16.7\%) in whom parasitaemia was detected on day 21 , while the remaining 5 patients $(83.4 \%)$ were

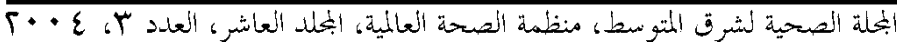




\begin{tabular}{|c|c|}
\hline Variable & Value \\
\hline Female (\%) & 35.5 \\
\hline Age (mean \pm SD years) & $6.9(3.9)$ \\
\hline $\begin{array}{l}\text { Duration of illness (mean } \pm \\
\text { SD days) }\end{array}$ & $4.6(3.8)$ \\
\hline Weight (mean \pm SD kg) & $21.1(9.1)$ \\
\hline Temperature (mean $\left.\pm \mathrm{SD}^{\circ} \mathrm{C}\right)$ & $37.9(1.3)$ \\
\hline Palpable spleen (\%) & 35.5 \\
\hline $\begin{array}{l}\text { Parasite count (mean } \pm \mathrm{SD} \\
\quad \text { rings } / \mu \mathrm{L})\end{array}$ & $8829.0(10011.3)$ \\
\hline $\begin{array}{l}\text { Temperature on day } 3 \\
\left(\text { mean } \pm S D{ }^{\circ} \mathrm{C}\right)\end{array}$ & $36.44(0.94)$ \\
\hline
\end{tabular}

$S D=$ standard deviation .

shown to have true recrudescence as confirmed by parasite genotyping using PCR techniques. There were no significant differences in the presenting characteristics between the responders and nonresponders to treatment.

Among the 31 patients who completed the study, gametocytes were found in 3 patients on day 0 . However, gametocytes were found on follow-up thick smears in 7 patients $(22.6 \%)$.

\section{Discussion}

The study was carried out in an area of high chloroquine resistance in New Halfa where no resistance to sulfadoxine/ pyrimethamine has been detected before [6]. The high level of sulfadoxine/pyrimetha-mine resistance in this study $(19 \%$ of patients) has not yet been reported in Sudan and is concerning. It may be explained by high use of the drug in this area. Our previous report of high chloroquine re- sistance and full efficacy of sulfadoxine/ pyrimethamine [6] has encouraged most practising doctors in the area to shift to sulfadoxine/pyrimethamine for the treatment of uncomplicated falciparum malaria even though it is still regarded as the second line for the treatment of uncomplicated malaria in Sudan. Another factor may be age; in this study treatment was assessed in children, who are more likely to be resistant to sulfadoxine/pyrimethamine than other age groups [3].

Surprisingly, the proportion of treatment failures (around $20 \%$ of patients) was similar to a study of Malawian children [16]. However, this comparison has to be taken cautiously. First, in Malawi sulfadoxine/pyrimethamine has been the first-line treatment since 1993, while in Sudan it is the second-line treatment for uncomplicated falciparum infection. Secondly, the Malawian study followed the 14-day protocol, whereas in the present study we followed the 28-day protocol. Thirdly, there are differences in malaria transmission in the 2 locations. Recently, sulfadoxine/pyrimethamine failures in Kenya were found to be $9.5 \%$ and $34.5 \%$ in 2 different areas at the same time; this difference was attributed to the differences in the endemicity, hence the increased drug pressure in this area [17].

In Kampala, Uganda, Kamya et al. found that the proportion of clinical and parasitological failures to sulfadoxine/pyrimethamine treatment were $11 \%$ and $30 \%$ respectively using the 28-day follow-up protocol [2]. However, they did not differentiate between true recrudescence and reinfection. Indeed most studies have followed the 14-day protocol [3], with the justification that cases with late parasitaemia after 14-35 days were likely to be new infections. However, most (83\%) of the patients with late parasitaemia in our

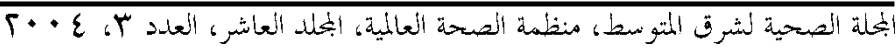


study were cases of recrudescence, as demonstrated and confirmed by parasite genotyping. Furthermore, very recently in same area, we found that parasites appearing on the 28th day following quinine treatment were due to true recrudescence and not reinfection [18].

Gametocytaemia was detected in $23 \%$ of the patients during the follow-up period. In our previous work, we noted fewer gametocytes in follow-up thick-film smears in Sudanese children given artemether compared with those given quinine [18]. The high level of gametocytaemia is a disadvantage of using sulfadoxine/ pyrimethamine alone, and therefore combining the treatment with artesunate may be one way of reducing resistance and gametocytaemia. Studies to confirm this are needed urgently.

On the basis of this decline in efficacy of sulfadoxine/pyrimethamine over 4 years in the same area, with most cases late treatment failures showing gametocytaemia and true recrudescence, we conclude that frequent/continuous assessment of antimalarial drugs is needed, using the 28-day rather than 14-day protocol and parasite genotyping. Combination chemotherapy with different regimens should be introduced in the area.

\section{Acknowledgements}

We wish to thank all the children and their families for their cooperation and we are very grateful to the local health authorities in Kassalsa State and to the Sudanese Sugar Company in New Halfa. Thanks are also extended to Mr Abdalla Ahmed Hufazalla and Mr Adil Amin for their excellent technical assistance.

\section{References}

1. Snow RW et al. Estimating mortality and morbidity and disability due to malaria among Africa's non-pregnant population. Bulletin of the World Health Organization, 1999, 77:624-40.

2. Kamya MR et al. The comparative efficacy of chloroquine and sulfadoxine-pyrimethamine for the treatment of uncomplicated falciparum malaria in Kampala, Uganda. Transactions of the Royal Society of Tropical Medicine and Hygiene, 2001, 95:50-5.

3. Schwobel B et al. Therapeutic efficacy of chloroquine plus sulphadoxine/pyrimethamine compared with monotherapy with either chloroquine or sulphadoxine/pyrimethamine in uncomplicated Plasmodium falciparum malaria in Laos. Tropical medicine and international health, 2003, 8:19-24.
4. Zucker JR et al. Childhood mortality during and after hospitalization in western Kenya: effect of malaria treatment regimens. American journal of tropical medicine and hygiene, 1996, 55:655-60.

5. Ibrahim AM et al. Assessment of chloroquine resistance of Plasmodium falciparum in children of Wad Medani (Central Sudan). Journal of tropical pediatrics, 1992, 38:162-6.

6. Adam I et al. In the Sudan: chloroquine resistance is worsening and quinine resistance is emerging. Sudan medical journal, 2001, 39:5-11.

7. Elkheir HK et al. Efficacy of sulfadoxine and pyrimethamine, doxycycline and their combination in the treatment of chloroquine resistant falciparum malaria. Saudi medical journal, 2001, 22: 690-3.

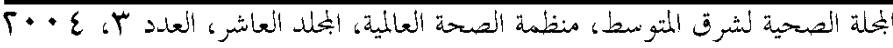


8. Ibrahim ME et al. A case of Plasmodium falciparum malaria sensitive to chloroquine but resistant to sulfadoxine/pyrimethamine combination in Sennar, Sudan. Transactions of the Royal Society of Tropical Medicine and Hygiene, 1991, 85:446.

9. Babiker HA et al. Genetics diversity of Plasmodium falciparum in a village in Eastern Sudan. 2. Drug resistance, molecular karyotypes and the mdr1 genotype of recent isolates. Transactions of the Royal Society of Tropical Medicine and Hygiene, 1991, 85:578-83.

10. Khalil IF. Sensitivity of chloroquine resistant Plasmodium falciparum to Fansimef, mefloquine and halofantrine in Gedaref, eastern Sudan [MSc thesis]. Khartoum, University of Khartoum, 1995.

11. El-Gadal AA. Malaria in the Sudan. In: Buck AA, ed. Proceedings of the conference on malaria in Africa. Washington DC, American Institute of Biological Sciences, 1986:156-9.

12. Himiedan YE et al. Anopheles arabiensis and insecticide resistance status in an irrigated area of eastern Sudan. Eastern Mediterranean health journal, 2004, 10(1\&2):167-74.

13. Severe falciparum malaria. World Health Organization, Communicable Disease
Cluster. Transactions of the Royal Society of Tropical Medicine and Hygiene, 2000, 94(suppl.1):S1-90.

14. Brockman A et al. Application of genetic markers to the identification of recrudescent Plasmodium falciparum infections on the northwestern border of Thailand. American journal of tropical medicine and hygiene, 1991, 60:14-20.

15. The use of antimalarial drugs. Report of an informed consultation. Geneva, World Health Organization, 2001 (WHO/CDS/ RBM/2001.33).

16. Macarthur JR et al. Efficacy of mefloquine and sulfadoxine-pyrimethamine for treatment of uncomplicated Plasmodium falciparum infection in Machinga District, Malawi, 1998. American journal of tropical medicine and hygiene, 2001, 65:679-84.

17. Dillen JV et al. A comparison of amodiaquine and sulfadoxine-pyrimethamine as first-line treatment of falciparum malaria in Kenya. Transactions of the Royal Society of Tropical Medicine and $\mathrm{Hy}$ giene, 1999, 93:185-8.

18. Adam I et al. Comparison of intramuscular artemether and intravenous quinine in the treatment of Sudanese children with severe falciparum malaria. East African medical journal, 2002, 79:621-5. 